09

\title{
Решетки населенностей, создаваемые парой униполярных аттосекундных импульсов в трехуровневой атомарной среде
}

\author{
(C) P.М. Архипов \\ Санкт-Петербургский государственный университет, \\ 199034 Санкт-Петербург, Россия \\ e-mail: arkhipovrostislav@gmail.com
}

Поступила в редакцию 08.07.2020 г.

В окончательной редакции 08.07.2020 г.

Принята к публикации 20.07.2020 г.

\begin{abstract}
Теоретически изучена возможность наведения решеток населенностей в трехуровневой резонасной среде с помощью пары униполярных аттосекундных импульсов, не перекрывающихся в среде. Показано, что результаты приближенного решения уравнения Шредингера согласуются с результатами численного решения системы уравнений для матрицы плотности трехуровневой среды, параметры которой близки к параметрам атомных паров рубидия.
\end{abstract}

Ключевые слова: аттосекундные импульсы, светоиндуцированные решетки.

DOI: $10.21883 /$ OS.2020.11.50177.186-20

\section{Введение}

В настоящее время экспериментально получены электромагнитные импульсы аттосекундной длительности $\left(1 \mathrm{as}=10^{-18} \mathrm{~s}\right)$ в экстремально ультрафиолетовом [1,2], оптическом [3-5] и рентгеновском спектральном диапазонах [6]. Они уже стали важной частью современной физики, так как с их помощью удается изучать движения электронов в атомах, молекулах и твердых телах, а также управлять этим движением [4,7]. Например, они используются для изучения динамики связанных электронов в атомах [4], в нанообъектах [8] и в туннельной микроскопии [9]. Параллельно обсуждается возможность получения униполярных субцикловых импульсов в оптике [10-12] и их применение для эффективного управления динамикой квантовых систем [13-15], ускорения заряженных частиц [16] и голографической записи [17].

Длительность аттосекундных импульсов может быть существенно меньше времен релаксации инверсии $T_{1}$ и поляризации $T_{2}$ среды, т. е. возможно когерентное взаимодействие импульсов с веществом [18]. При когерентном взаимодействии предельно коротких импульсов со средой они могут быстро менять населенности атомных уровней за времена порядка длительности импульса. При этом, как показано ранее, последовательность аттосекундных импульсов, не перекрывающихся в среде, может наводить в среде решетки населеностей, а в случае действия на среду трех и более импульсов появляется возможность стирания решеток и мультиплицирования их пространственной частоты [19-22]. В традиционном же подходе создание светоиндуцированных решеток происходит при интерференции двух длинных квазимонохроматических пучков, перекрывающихся в среде [23]. Такие решетки находят многочисленные применения в спектроскопии, так как дифракция пробного излучения на них используется, например, для изучения свойств веществ и измерения их оптических характеристик [23]. Возможность создания решеток в среде с помощью пары импульсов, которые не встречаются в среде, была продемонстрирована экспериментально довольно-таки давно в случае длинных наносекундных импульсов, см. работы $[24,25]$ и обзор [26] и цитируемую литературу. Впоследствии данная возможность была изучена теоретически в двухуровневой среде в случае биполярных одноцикловых [19-20] и униполярных субцикловых аттосекундных импульсов [21-22]. В работе [27] с помощью приближенного решения уравнения Шредингера на основе теории возмущений данный результат был обобщен на случай многоуровневых сред, возбуждаемых субцикловыми импульсами. В последующих работах $[28,29]$ была показана возможность селективного воздействия на населенности квантовых систем с помощью последовательности униполярных аттосекундных импульсов за счет изменения задержки между ними. Данное обстоятельство открывает новые возможности в оптике, так как дает возможность управления динамикой атомных систем с помощью униполярных аттосекундных импульсов.

В настоящей работе, которая является продолжением предыдущих исследований, на основе численого решения системы уравнений для матрицы плотности рассматривается возможность наведения решеток в трехуровневой резонасной среде с помощью пары аттосекундных униполярных импульсов, не перекрывающихся в среде. Параметры трехуровневой среды близки по значениям к резонасным переходам в парах рубидия ${ }^{87} \mathrm{Rb}$. Показано, что результаты численных расчетов качественно согласуются с результами аналитических расчетов, проведенных на основе приближенных аналитических вы- 


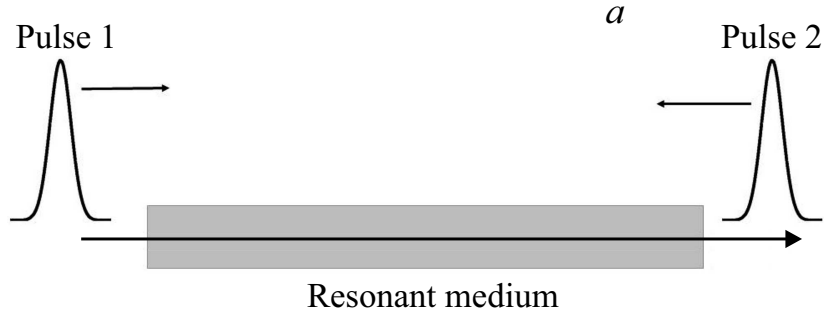

$b$

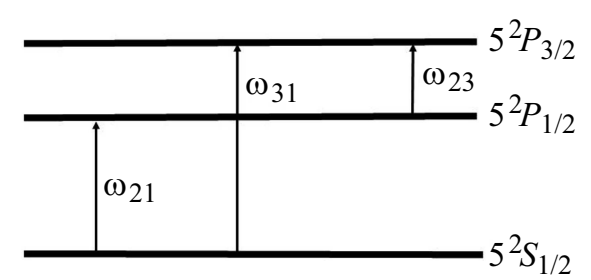

Рис. 1. (a) Схема создания решеток населеностей в трехуровневой среде с помощью пары субцикловых аттосекундных импульсов, $(b)$ схема рабочих уровней среды (в качестве примера для простоты указаны термы рубидия).

ражений, полученных ранее в [27-29] на основе теории возмущений.

\section{Наведение решеток населенностей с помощью пары униполярных аттосекундных импульсов в трехуровневой среде}

Схема создания решеток в трехуровневой среде и конфигурация уровней среды представлены на рис. 1. Данная схема создания решеток была рассмотрена ранее в $[19-22,27]$. Разреженная среда из атомных паров возбуждается парой аттосекундных импульсов. Излучение считаем линейно поляризованным, с плоским волновым фронтом, распространяющимся вдоль оси $z$. При этом первый импульс распространяется в среде слева направо. А второй импульс распространяется в среде справа налево, спустя интервал времени короче времен релаксации в среде, и после того как первый импульс покинул среду. В такой схеме опыта импульсы не встречаются в среде и взаимодействуют со средой когерентно. Плотность атомных паров считаем малой, чтобы изменением формы импульсов при распространении можно было пренебречь.

В данной схеме опыта первый импульс оставляет после себя квантовую систему в суперпозиционном состоянии. Когерентность среды (поляризация и недиагональные элементы матрицы плотности) осциллирует на частотах резонансных переходов, причем фаза этих осцилляций зависит от координаты $z$. Затем второй импульс взаимодействует с наведенной поляризацией среды. Так как фаза ее осцилляций зависит от координаты $z$, то результат взаимодействия со вторым импульсом будет зависеть от задержки между импульсами, т.е. в среде возникнет гармоническая решетка населенностей на каждом резонансном переходе среды [19-22,27-29].

Среда моделируется в трехуровневом приближении (рис. $1, b$ ). Будем рассматривать D-линии рубидия с длинами волн переходов 794.766 и $780.032 \mathrm{~nm}$ [30]. Переход 23 считаем слабым и для простоты его дипольный момент положим равным 0.

Пусть на среду воздействует пара субцикловых униполярных импульсов гауссовой формы, имеющих в общем виде разные амплитуды и длительности $\tau_{1}$ и $\tau_{2}$ :

$$
E(t)=E_{1} \exp \left[-t^{2} / \tau_{1}^{2}\right]+E_{2} \exp \left[-(t-\Delta)^{2} / \tau_{2}^{2}\right]
$$

$(\Delta-$ задержка между импульсами). Получаемые на практике субцикловые аттосекундные импульсы содержат мощную полуволну поля одной полярности и длинный затухающий хвост противоположной полярности с малой амплитудой [3-5]. Как показывают результаты исследований $[28,29]$, влиянием хвоста можно пренебречь, если его длина намного превосходит длину основного импульса, а амплитуда существенно меньше амплитуды униполярной полуволны. Поэтому в дальнейшем для простоты мы будем рассматривать действие на систему двух униполярных импульсов (1), а влиянием хвостов пренебрежем.

В работах $[28,29]$ на основании приближенного решения уравнения Шредингера в первом порядке теории возмущений было получено следующее выражение для вероятности переходов под действием пары гауссовых импульсов (1):

$$
\begin{aligned}
W_{1 k} & =\frac{d_{1 k}^{2}}{\hbar^{2}} S_{E_{1}}^{2} \exp \left[-\frac{\omega_{1 k}^{2} \tau_{1}^{2}}{2}\right]+\frac{d_{1 k}^{2}}{\hbar^{2}} S_{E_{2}}^{2} \exp \left[-\frac{\omega_{1 k}^{2} \tau_{2}^{2}}{2}\right] \\
& +2 \frac{d_{1 k}^{2}}{\hbar^{2}} S_{E_{1}} S_{E_{2}} \exp \left[-\omega_{1 k}^{2}\left(\tau_{1}^{2}+\tau_{2}^{2}\right) / 4\right] \cos \left(\omega_{1 k} \Delta\right)
\end{aligned}
$$

$\left(S_{E 1}\right.$ и $S_{E 2}-$ электрические площади импульсов, $\left.S_{E} \equiv \int E(t) d t\right)$. В случае действия на систему двух одинаковых импульсов $\left(S_{E 1}=S_{E 2}=S_{E}, \tau_{1}=\tau_{2}=\tau\right)$ выражение (2) упрощается

$$
w_{1 k}=2 \frac{d_{1 k}}{\hbar^{2}} S_{E}^{2} \exp \left[-\frac{\omega_{1 k}^{2} \tau^{2}}{2}\right]\left(1+\cos \left(\omega_{1 k} \Delta\right)\right) .
$$

Из (2),(3) видна периодическая зависимость вероятности переходов от задержки между импульсами. Таким образом, в случае протяженной среды пара субцикловых импульсов создает на резонансных переходах среды гармоническую решетку населенностей, глубина модуляции которой зависит от электрической площади импульса. А в случае тонкого слоя среды (единичного атома) формулы (2), (3) иллюстрируют возможности селективного воздействия на резонансные переходы в среде с помощью пары импульсов путем изменения задержки между импульсами $[28,29]$. 
Параметры модели, используемые в расчетах

\begin{tabular}{|c|c|}
\hline Амплитуда аттосекундных импульсов возбуждения & $E_{0}=10^{7} \mathrm{~V} / \mathrm{cm}$ \\
\hline Длительность импульсов возбуждения & $\tau=500$ as \\
\hline $\begin{array}{l}\text { Частота перехода } 12 \\
\text { (длина волны перехода) }\end{array}$ & $\begin{array}{c}\omega_{21}=2.4 \cdot 10^{15} \mathrm{rad} / \mathrm{s} \\
\left(\lambda_{21}=794.766 \mathrm{~mm}\right)\end{array}$ \\
\hline Дипольный момент перехода 12 & $d_{12}=7.6 \mathrm{D}$ \\
\hline $\begin{array}{l}\text { Частота перехода } 13 \\
\text { (длина волны перехода) }\end{array}$ & $\begin{array}{c}\omega_{31}=2.4 \cdot 10^{15} \mathrm{rad} / \mathrm{s} \\
\left(\lambda_{31}=780.032 \mathrm{~mm}\right)\end{array}$ \\
\hline Дипольный момент перехода 13 & $d_{13}=10.7 \mathrm{D}$ \\
\hline Частота перехода 23 & $\omega_{23}=4.48 \cdot 10^{13} \mathrm{rad} / \mathrm{s}$ \\
\hline Дипольный момент перехода 23 & $d_{23}=0 \mathrm{D}$ \\
\hline
\end{tabular}

Для проверки формулы (3) нами проведены численные расчеты для трехуровневой среды, взаимодействующей с парой гауссовых импульсов (1). Взаимодействие трехуровневой среды с электрическим полем субцикловых импульсов подчиняется следующей системе уравнений для элементов матрицы плотности, записанной без приближения меняющихся амплитуд и вращающейся волны [31]:

$$
\begin{gathered}
\frac{\partial}{\partial t} \rho_{21}=-i \omega_{21} \rho_{21}-i \frac{d_{12}}{\hbar} E\left(\rho_{22}-\rho_{11}\right) \\
-i \frac{d_{13}}{\hbar} E \rho_{23}+i \frac{d_{23}}{\hbar} E \rho_{31}, \\
\frac{\partial}{\partial t} \rho_{32}=-i \omega_{23} \rho_{32}-i \frac{d_{23}}{\hbar} E\left(\rho_{33}-\rho_{22}\right) \\
-i \frac{d_{12}}{\hbar} E \rho_{31}+i \frac{d_{13}}{\hbar} E \rho_{21}, \\
\frac{\partial}{\partial t} \rho_{31}=-i \omega_{31} \rho_{31}-i \frac{d_{13}}{\hbar} E\left(\rho_{33}-\rho_{11}\right) \\
\frac{\partial}{\partial t} \rho_{11}=i \frac{d_{12}}{\hbar} E \rho_{32}+i \frac{d_{23}}{\hbar} E \rho_{21}, \\
\frac{\partial}{\partial t} \rho_{22}=-i \frac{d_{12}}{\hbar} E\left(\rho_{21}-\rho_{21}^{*}\right)-i \frac{d_{13}}{\hbar} E\left(\rho_{13}-\rho_{13}^{*}\right), \\
\frac{\partial}{\partial t} \rho_{33}=i \frac{d_{13}}{\hbar} E\left(\rho_{13}-\rho_{13}^{*}\right)+i \frac{d_{23}}{\hbar} E\left(\rho_{23}-\rho_{23}^{*}\right)
\end{gathered}
$$

Уравнения (4)-(6) описывают эволюцию недиагональных элементов матрицы плотности $\rho_{21}, \rho_{32}, \rho_{31}$, определяющих динамику поляризации среды. Уравнения (7), (8) описывают эволюцию диагональных элементов матрицы плотности $\rho_{11}, \rho_{22}, \rho_{33}$, имеющих смысл населенностей 1-го, 2-го и 3-го состояний среды соответственно. Остальные параметры: $d_{12}, d_{13}, d_{23}-$ дипольные моменты переходов, $\omega_{21}, \omega_{23}, \omega_{31}$ - частоты переходов $\hbar-$ приведенная постоянная Планка.
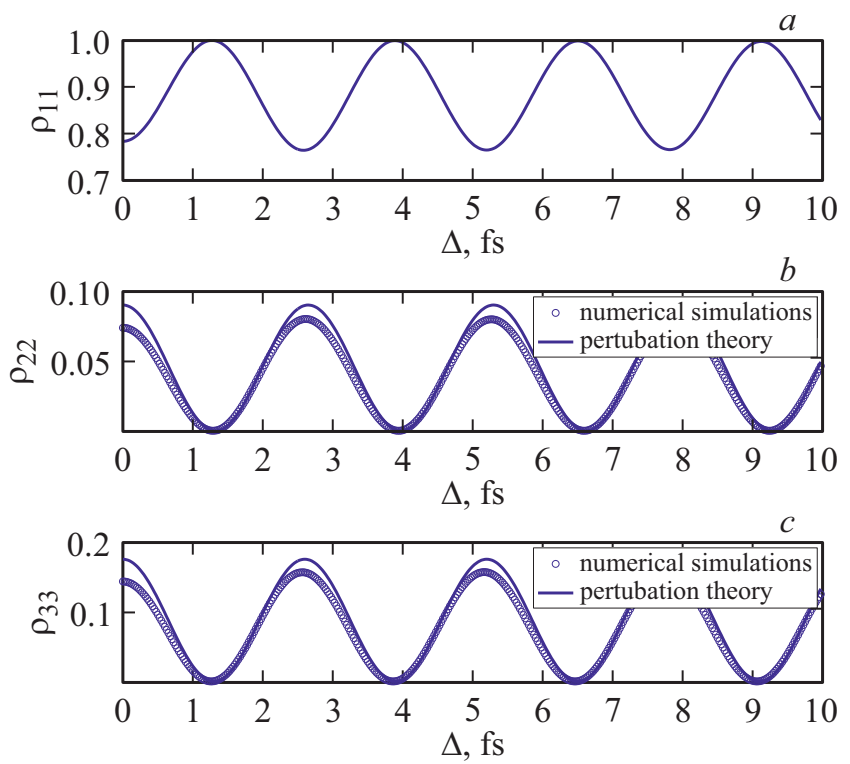

Рис. 2. Зависимости населенности уровней $\rho_{11}, \rho_{22}, \rho_{33}$ от задержки между импульсами $\Delta$. Кружками показаны результаты, полученные на основании численного решения системы уравнений (4)-(9), сплошная линия иллюстрирует значения, рассчитанные аналитически с помощью теории возмущений по формуле (2).

Релаксационными членами пренебрегаем ввиду малой длительности импульсов.

Для каждого значения задержки $\Delta$ между импульсами из заданного интервала система уравнений (4)-(9) интегрировалась численно при значениях параметров задачи, приведенных в таблице, и запоминалось значение населенностей каждого уровня после окончания действия импульсов. Длительность импульсов возбуждения принималась равной 500 as, что близко к экспериментально достижимым значениям [4].

Рис. 2 иллюстрирует зависимости населенностей уровней $\rho_{11}, \rho_{22}, \rho_{33}$ как функции задержки $\Delta$, рассчитанные с помощью численного решения системы уравнений (4)- 

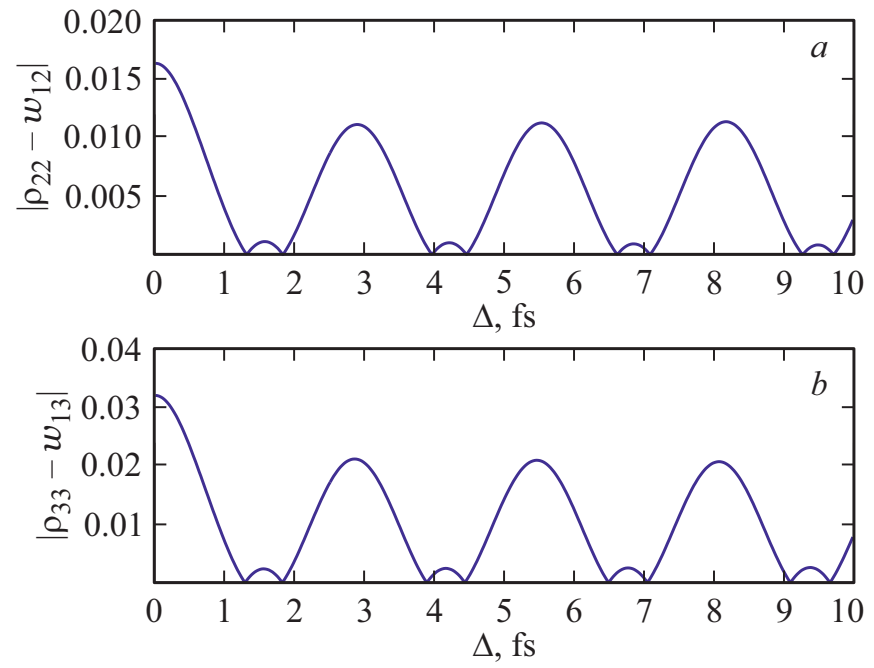

Рис. 3. Зависимости абсолютной величины разницы между значениями населенности 2-го $\left|\rho_{22}-w_{12}\right|(a)$ и 3-го уровня $\left|\rho_{33}-w_{13}\right|(b)$ от задержки между импульсами $\Delta$, рассчитанные численно и аналитически по фомуле (2).

(9) (кружки), и значения населенностей, полученные аналитически (сплошная линия) по теории возмущений с помощью формулы (2). Видна гармоническая зависимость населенностей уровней от задержки между импульсами, что говорит о создании периодических решеток населенностей в случае протяженной среды. В случае тонкого слоя среды (единичного атома) данный результат показывает возможность селективного воздействия (возбуждения-девозбуждения) квантовых переходов среды с помощью изменения задержки между ипульсами $[28,29]$. Данная ситуация имеет аналогию в классической механике, когда колебаниями качающихся качелей (маятника) можно управлять с помощью короткого толчка (в зависимости от фазы их колебаний толчком можно, например, либо остановить качели, либо, наоборот, раскачать их сильнее). В нашем случае первый субцикловый импульс оставляет после себя систему в суперпозиционном состоянии, и когерентность среды осциллирует на частотах переходов. А второй импульс в зависимости от задержки может управлять осцилляциями этой когерентности, тем самым управляя населенностями квантовых переходов среды.

Также из рис. 2 видно, что результаты численного расчета неплохо согласуются с результами вычислений, полученными на основе приближенной аналитической формулы (2).

Рис. 3 иллюстрирует разницу между значениями $\left|\rho_{22}-w_{12}\right|$ и $\left|\rho_{33}-w_{13}\right|$, полученными на основе численного расчета и с помощью аналитического выражения (2). Видно, что разница между результатами, полученными аналитически и численно, составляет порядка $1-2 \%$.

\section{Заключение}

В работе на основании численного решения системы уравнений для матрицы плотности трехуровневой системы показана возможность создания гармоничеких решеток населенностей с помощью пары субцикловых аттосекундных импульсов, не перекрывающихся в протяженной среде. Частоты переходов и дипольные моменты трехуровневой среды близки по значениям к параметрам паров рубидия ${ }^{87} \mathrm{Rb}$. Показано, что результаты численного расчета неплохо согласуются с результатами, полученными аналитически на основе приближенного решения уравнения Шредингера в первом порядке теории возмущений. Отклонения в значении населенностей, полученные численно и аналитически, составляют порядка $1 \%$.

В случае тонкого слоя среды (единичного атома) данные результаты показывают возможность селективного воздействия на квантовые переходы в среде с помощью изменения задержки между импульсами.

Исследованный эффект может использоваться для сверхбыстрого управления свойствами сред с помощью аттосекундных импульсов [19-22,27-29]. Дифракция пробного излучения на индуцированных решетках населенностей может быть использована для измерения времени релаксации поляризации среды [25]. Также изученные решетки могут найти применение в голографии с помощью субцикловых импульсов без взаимной когерентности между опорным и предметным пучками [17].

\section{Благодарности}

Автор выражает благодарность своим коллегам М.В. Архипову и Н.Н. Розанову за полезные обсуждения и ценные замечания, способствовавшие улучшению данной работы.

\section{Финансирование работы}

Исследования выполнены при финансовой поддержке РНФ в рамках научного проекта № 19-72-00012.

\section{Конфликт интересов}

Автор заявляет, что у него нет конфликта интересов.

\section{Список литературы}

[1] Krausz F., Ivanov M // Rev. Mod. Phys. 2009. V. 81. P. 163.

[2] Calegari F. et al. // J. Phys. B: Atomic, Molecular and Optical Physics. 2016. V. 49. N 6. P. 062001.

[3] Wu H.-C. , Meyer-ter-Vehn J. // Nature Photon. 2012. V. 6. P. 304.

[4] Hassan M.T., Luu T.T., Moulet A., Raskazovskaya O. et al. // Nature. 2016. V. 530. P. 66.

[5] Xu J., Shen B., Zhang X. et al. // Sci. Rep. 2018. V. 8. P. 2669. 
[6] Mak A., Shamuilov G., Sal ?en P., Dunning D., Hebling J., Kida Y., Kinjo R., McNeil B.W., Tanaka T., Thompson N. et al. // Reports on Progress in Physics. 2019. V. 82. P. 025901.

[7] Ramasesh K., Leone S.R., Neumark D.M. // Annu. Rev. Phys. Chem. 2016. V. 67. P. 41.

[8] Krüger M., Lemell C., Wachter G., Burgdörfer J., Hommelhoff P. // J. Phys. B: Atomic, Molecular and Optical Physics. 2018. V. 51. N 17. P. 172001.

[9] Garg M., Kern K. // Science. 2020. V. 367. N 6476. P. 411415.

[10] Архипов Р.М., Пахомов А.В., Архипов М.В., Бабушкин И., Толмачев Ю.А., Розанов Н.Н // Письма в ЖЭТФ. 2017. T. 105. № 6. C. 388-400; Arkhipov R.M., Pakhomov A.V., Babushkin I., Tolmachev Yu.A., Rosanov N.N. // JETP Lett. 2017. V. 105. N 6. P. 408-418.

[11] Архипов Р.М., Архипов М.В., Шимко А.А., Пахомов А.В., Розанов Н.Н // Письма в ЖЭТФ. 2019. Т. 110. № 10. C. 9-20; Arkhipov R.M., Arkhipov M.V., Shimko A.A., Pakhomov A.V., Rosanov N.N. // JETP Lett. 2019. V. 110. N 1. P. $15-24$.

[12] Архипов Р.М, Архипов М.В, Розанов Н.Н. // Квант. электрон. 2020, 50(9), C. 801-815; Arkhipov R.M., Arkhipov M.V; Rosanov N.N // Quantum Electron. 2020, 50(9), P. 801-815.

[13] Chai X., Ropagnol X., Raeis-Zadeh S.M., Reid M., SafaviNaeini S., Ozaki T. // Phys. Rev. Lett. 2018. V. 121. N 14. P. 143901.

[14] Розанов Н.Н // Опт. и спектр. 2018. Т. 124. № 1. С. 75-77; Rosanov N.N. // Opt. Spectr. 2018. V. 124. № T1. P. 72-74.

[15] Arkhipov R.M., Arkhipov M.V., Babushkin I., Demircan A., Morgner U., Rosanov N.N. // Opt. Lett. 2019. V. 44. N 5. P. 1202.

[16] Розанов Н.Н., Высотина Н.В. // ЖЭТФ. 2020. Т. 157. № 1. C. 63-66; Rosanov N.N., Vysotin N.V. // JETP. 2020. V. 130. N 1. P. 52-55.

[17] Архипов Р.М., Архипов М.В., Розанов Н.Н. // Письма в ЖЭТФ. 2020. Т. 111. В. 9. С. 586 - 590; Arkhipov R.M., Arkhipov M.V., Rosanov N.N. // JETP. Lett. 2020. V. 111. N 9. P. 484-488.

[18] Аллен Л., Эберли Дж. Оптический резонанс и двухуровневые атомы. М.: Мир, 1978; Allen L., Eberly J.H. Optical resonance and two-level atoms. NY:: Wiley, 1975.

[19] Arkhipov R.M., Arkhipov M.V., Babushkin I.V., Demircan A., Morgne U., Rosanov N.N // Opt. Lett. 2016. V. 41. P. 4983.

[20] Arkhipov R.M., Pakhomov A.V., Arkhipov M.V., Babushkin I., Demircan A., Morgner U., Rosanov N.N. // Sci. Rep. 2017. V. 7(1). P. 12467.

[21] Архипов Р.М., Архипов М.В., Пахомов А.В., Бабушкин И., Розанов Н.Н. // Квант. электрон. 2017. Т. 47. № 7. С. 589; Arkhipov R.M., Arkhipov M.V., Babushki I., Pakhomov A.V., Rosanov N.N. // Quantum Electronics. 2017. V 47. N 7. P. 589.

[22] Arkhipov R.M., Arkhipov M.V., Pakhomov A.V., Babushkin I., Rosanov N.N. // Las. Phys. Lett. 2017. V. 14. N 9. P. 1.

[23] Eichler H.J., Günter P., Pohl D.W. Laser-Induced Dynamic Gratings. Berlin, Heidelberg, N Y., Tokyo: Springer-Verlag, 1981.

[24] Abella I.D., Kurnit N.A., Hartmann S.R. // Phys. Rev. 1966. V. 141. P. 391.
[25] Штырков Е.И., Лобков В.С., Ярмухаметов Н.Г. // Письма в ЖЭТФ. 1978. Т. 27. № 12. С. 685; Shtyrko E.I., Lobkov V.S., Yarmukhametov N.G. // JETP Lett. 1978. V. 27. N 12. P. 648 (1978).

[26] Штырков Е.И. // Опт. и спектр. 2013. Т. 114. № 1. С. 105; Shtyrkov E.I. // Opt. Spectrosc. 2013. V. 114. N 1. P. 96.

[27] Архипов Р.М., Архипов М.В., Пахомов А.В., Розанов Н.Н. // Квант. электрон. 2019. Т. 49. С. 958; Arkhipov R.M., Arkhipov M.V., Pakhomov A.V., Rosanov N.N. // Quantum Electronics. 2019. V. 49. P. 958.

[28] Архипов Р.М., Архипов М.В., Пахомов А.В., Розанов Н.Н. // Опт. и спектр. 2020. Т. 128. С. 106; Arkhipov R.M., Arkhipov M.V., Pakhomov A.V., Rosanov N.N. // Opt. Spectrosc. 2020. V. 128. P. 102.

[29] Arkhipov R., Pakhomov A., Arkhipov M., Demircan A., Morgner U., Rosanov N., Babushkin I. // Optics Express. 2020. V. 28. N 11. P. 17020-17034.

[30] Steck D.A., http://steck.us/alkalidata (revision 2.1.1) (2009).

[31] Розанов Н.Н. Диссипативные оптические солитоны от микро- к нано- и атто-. М.: Физматлит, 2011. 